\title{
Contrast Mechanisms in $\mathrm{Ga}^{+}$Ion Induced Secondary Electron Images
}

\author{
Lucille A. Giannuzzi ${ }^{*}$ and M. Utlaut ${ }^{* *}$ \\ *FEI Company, 5350 NE Dawson Creek Drive, Hillsboro, OR USA 97124 \\ *** Department of Physics, University of Portland, Portland, Oregon USA 97203
}

Any particle induced electron emission may be characterized by a 3 step process: (i) the creation of electrons, (ii) transport or diffusion of electrons within the solid, and (iii) escape of electrons over the surface-vacuum barrier [1]. It has generally been accepted that steps (ii) and (iii) are common to all electron emission processes regardless of the bombarding particle (e.g., ions or electrons). The first step or generation of electrons in a solid by energetic ions may have contributions due to both potential energy emission (PE) and kinetic energy (KE) emission processes. PE contributions are due to fast electronic transitions from the surface into empty projectile states and are more prevalent as the charge state of the ion increases. Thus, the PE contributions are generally considered to be negligible for singly charged ions and at energies such as those used in $\mathrm{Ga}^{+}$focused ion beam (FIB) instruments, and therefore, the SE emission process due to $\mathrm{Ga}^{+}$bombardment will be dominated by $\mathrm{KE}$ effects. KE contributions are due to the transfer of conservation of energy and momentum of the energetic projectile into the solid. The KE contributions are proportional to the total stopping power (i.e., nuclear + electronic) of the ion-solid interactions which is a non-monotonic function for $\mathrm{Ga}^{+}$ across the periodic table as shown in FIG. 1. It should be noted that for typical $\mathrm{Ga}^{+} \mathrm{FIB}$ operating conditions, nuclear stopping dominates the ion-solid interaction process. The sputter yield (atoms/ion) for $30 \mathrm{keV} \mathrm{Ga}{ }^{+}$at $0^{\circ}$ incident angle is also plotted as a function of atomic number in FIG. 1. Note that while the sputter yield is also non-monotonic across the periodic table, the sputter yield and stopping power do not follow the same periodic trends.

Previous experimental work has also shown that $30 \mathrm{keV} \mathrm{Ga}^{+}$ion induced SE (ISE) contrast is nonmonotonic as a function of atomic number $[2,3]$. This observed ISE contrast is consistent with the non-monotonic behavior of ion-solid interaction theory. However, the observed ISE contrast is not directly proportional to the stopping power as presumed by theory [1]. A target will be milled during the $\mathrm{Ga}^{+}$FIB imaging process according to its sputter yield. Thus, a proper model for secondary electron escape must account for a moving surface toward the electrons that are being generated in the target. Therefore, the sputter yield can influence the diffusion length of the electron transport defined by step (ii) above. FIG. 2 shows ISE contrast for previously reported results $[2,3]$ and a normalized function of the sputter yield and stopping power $(\mathrm{dE} / \mathrm{dx})$ showing that the ISE contrast more closely fits the behavior when the affects from both of these ion-solid interactions are considered. Future work will incorporate Monte Carlo simulations to assess the ISE contrast mechanisms [4].

\section{References}

[1] Slow Heavy-Particle Induced Electron Emission from Solid Surfaces, eds. Hannspeter Winter and Joachim Burgdorfer, Springer-Verlag, Berlin, (2007).

[2] Lucille A. Giannuzzi et al., Microsc Microanal 14(Suppl 2), (2008), 1188.

[3] Lucille A. Giannuzzi et al., M. Luysberg, K. Tillmann, T. Weirich (Eds.): EMC 2008, Vol. 1: Instrumentation and Methods, (C) Springer-Verlag Berlin Heidelberg (2008) 665.

[4] Thanks for Joe Michael, Sandia National Labs, for helpful discussions. 


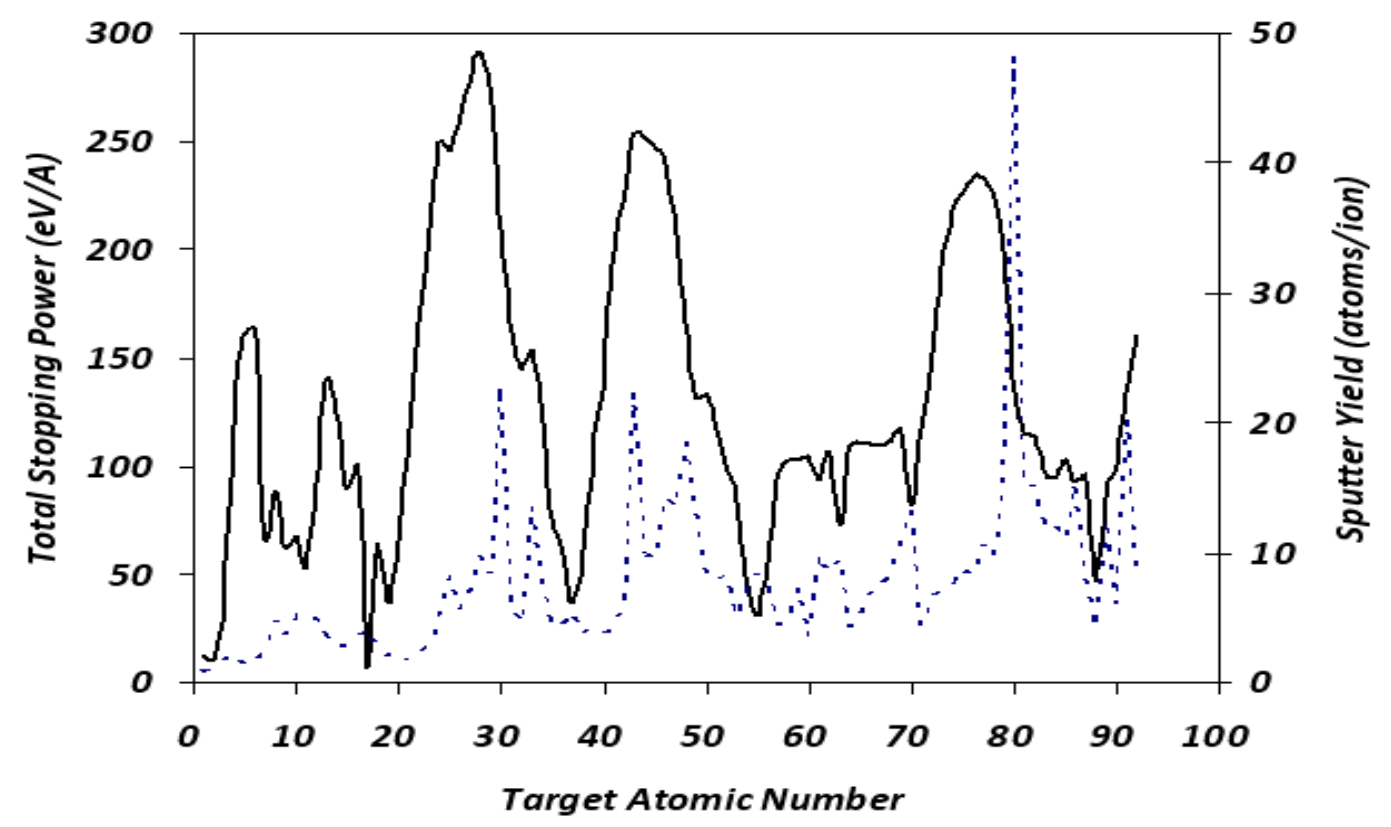

total stopping power - . - Y Y $(0 \mathrm{deg})$

FIG. 1. The total stopping power and sputter yield for $\mathrm{Ga}^{+}$as a function of atomic number for all the elements of the periodic table. Each function shows non-monotonic behavior and often dissimilar characteristics.

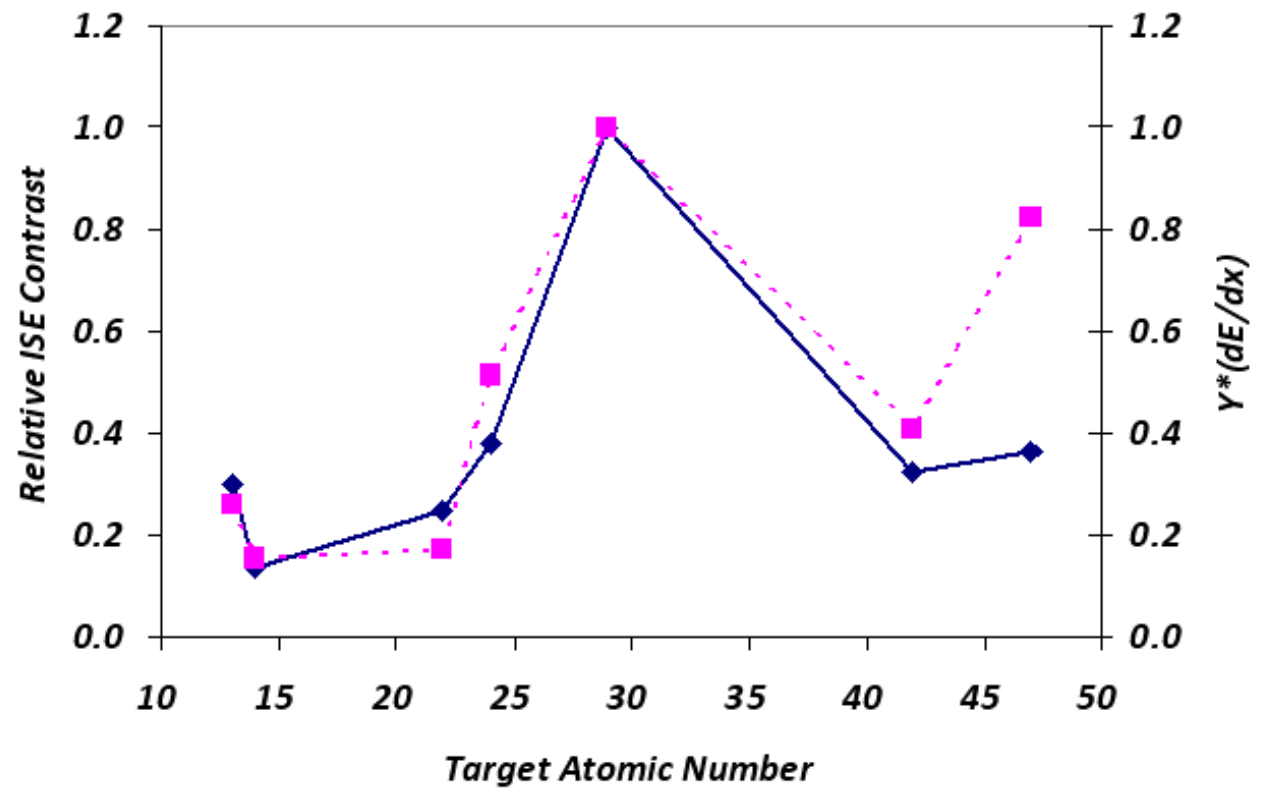

$$
\longrightarrow-I S E--Y^{*}(d E / d x)
$$

FIG. 2. Observed ISE contrast and qualitative comparison of $\mathrm{Y}^{*}(\mathrm{dE} / \mathrm{dx})$. 